\title{
The stability of the resonance set for a problem with jumping non-linearity
}

\section{Marcel d'Aujourd'hui}

Département de Mathématiques, EPFL, CH-1015, Lausanne, Switzerland

(MS received 9 October 1987. Revised MS received 29 April 1987)

\section{Synopsis}

For $Q \in L^{2}(0,1)$ we investigate the set $\Gamma \in \mathbb{R}^{2}$ of pairs $(\alpha, \beta)$ for which the problem

$$
\int_{0}^{1} u^{\prime}(x) v^{\prime}(x) d x-\int_{0}^{1}(u(x) v(x))^{\prime} Q(x) d x=\int_{0}^{1}\left(\alpha u(x)^{+}-\beta u(x)^{-}\right) v(x) d x
$$

$\forall v \in H_{0}^{1}(0,1)$ has a nontrivial solution $u \in H_{0}^{1}(0,1)$ which has exactly one zero in $(0,1)$ and is positive near $x=0$. We show that $\Gamma$ is stable in a certain sense under small perturbations of $Q$. The dependence of $\Gamma$ upon $Q$ is illustrated by an example.

\section{Introduction}

In recent years many authors have studied nonlinear problems of the type

$$
T u=f(u)+h,
$$

where $T: D(T) \subset L^{2}(\Omega) \rightarrow L^{2}(\Omega)$ is a self-adjoint operator with compact resolvent in $L^{2}(\Omega), \Omega$ an open domain of $\mathbb{R}^{N}$ and $f: \mathbb{R} \rightarrow \mathbb{R}$ a continuous function such that the following limits exist:

$$
\lim _{s \rightarrow+\infty} \frac{f(s)}{s}=\alpha \text { and } \lim _{s \rightarrow-\infty} \frac{f(s)}{s}=\beta .
$$

The set of all $h \in L^{2}(\Omega)$ such that (1) has solution depends upon the values of $\alpha$ and $\beta$.

Among the numerous results obtained for this question we recall that of $T$. Gallouët and O. Kavian [1,2] and B. Ruf [3]. Let $\sigma=\left\{\lambda_{i}: \lambda_{i}<\lambda_{i+1} \forall i \in \mathbb{N}\right\}$ be the spectrum of $T$. Suppose that $\lambda_{k}$ is a simple eigenvalue with $k \geqq 2$ and let $\psi_{k}$ be an eigenfunction corresponding to $\lambda_{k}$ such that $\int_{\Omega} \psi_{k}^{2}=1$. Then for $(\alpha, \beta) \epsilon$ $\left(\lambda_{k-1}, \lambda_{k+1}\right) \times\left(\lambda_{k-1}, \lambda_{k+1}\right)=S_{k}$ there exists a unique pair $(u, C) \in D(T) \times \mathbb{R}$ such that

$$
\begin{aligned}
& \left.\begin{array}{l}
T u=\alpha u^{+}-\beta u^{-}+C \psi_{k} \\
\int_{\Omega} u \psi_{k}=1
\end{array}\right\}
\end{aligned}
$$

where $s^{+}=\max \{s, 0\}$ and $s^{-}=\max \{-s, 0\}$. The function $C: S_{k} \rightarrow \mathbb{R}$ so defined is continuous and strictly decreasing with respect to each of the variables $\alpha$ and $\beta$. In particular $\Gamma=\left\{(\alpha, \beta) \in S_{k}: C(\alpha, \beta)=0\right\}$ is a continuous curve passing through $\left(\lambda_{k}, \lambda_{k}\right)$ and it is the graph of a strictly decreasing function $\eta(\alpha)$. The curve $\Gamma$ splits $S_{k}$ into two connected components. For $(\alpha, \beta) \in S_{k}$ the following holds. If 
$C(\alpha, \beta) C(\beta, \alpha)>0$, then for all $h \in L^{2}(\Omega)$ (1) has a solution. If $C(\alpha, \beta) C(\beta, \alpha)<0$, there exists $h \in L^{2}(\Omega)$ such that (1) has no solution.

In [4] C. A. Stuart has shown that if $\Omega \subset \mathbb{R}^{N}$ is bounded with smooth boundary, $T=-\Delta$ and $D(T)=W^{2, p}(\Omega) \cap W^{1, p}(\Omega)$ for some $p>N / 2$, then $C$ is a $C^{1}$-function on $S_{k}$. He gives formulae for $\partial C / \partial \alpha, \partial C / \partial \beta$ and $\eta^{\prime}(\alpha)$.

Note that the normalisation in (2) enables us to distinguish the two symmetric curves $C(\alpha, \beta)=0$ and $C(\beta, \alpha)=0$. In fact if $u$ is a solution of (2) with $C=0$, then $v=-u$ solves $T v=\beta v^{+}-\alpha v^{-}$and $\int_{\Omega} v \psi_{k}=-1$.

The result of $[1,2]$ and $[3]$ motivates a more precise study of $\Gamma$. In this paper we consider the following problem: given $Q \in L^{2}(0,1)$, describe the set of pairs $(\alpha, \beta)$ such that

$$
\int_{0}^{1} u^{\prime}(x) v^{\prime}(x) d x-\int_{0}^{1}(u(x) v(x))^{\prime} Q(x) d x=\int_{0}^{1}\left(\alpha u(x)^{+}-\beta u(x)^{-}\right) v(x) d x
$$

for all $v \in H_{0}^{1}(0,1)$ has a nontrivial solution $u \in H_{0}^{1}(0,1)$ which has exactly one zero in $(0,1)$.

If $Q \in C^{\mathbf{1}}([0,1])$, then (3) reduces to

$$
\left.\begin{array}{l}
-u^{\prime \prime}(x)+q(x) u(x)=\alpha u(x)^{+}-\beta u(x)^{-} \\
u(0)=u(1)=0
\end{array}\right\}
$$

where $q(x)=Q^{\prime}(x)$ for all $x \in[0,1]$. As we shall see, the solution of (3) can be normalised as in (2) so that we are concerned with a problem of type (2) with $C=0$ and $k=2$. The unique zero $z \in(0,1)$ of the solution of (3) splits the equation (3) into two linear problems

$$
\begin{aligned}
& \int_{0}^{z} u^{\prime}(x) v^{\prime}(x) d x-\int_{0}^{z}(u(x) v(x))^{\prime} Q(x) d x=\alpha \int_{0}^{z} u(x) v(x) d x \forall v \in H_{0}^{1}(0, z) \\
& \int_{z}^{1} u^{\prime}(x) v^{\prime}(x) d x-\int_{z}^{1}(u(x) v(x))^{\prime} Q(x) d x=\beta \int_{z}^{1} u(x) v(x) d x \forall v \in H_{0}^{1}(z, 1) .
\end{aligned}
$$

These are studied in the next section. The first eigenvalues of (5) and (6), the only ones we are interested in, are shown to be stable under small perturbations of $z$ and $Q$. In Section $3 \Gamma$ is constructed using $z$ as parameter. It is proved that $\Gamma$ is stable in a certain sense under small perturbations of $Q$. In Section 4 an example illustrates the effect of $Q$ on $\Gamma$. In particular $\Gamma$ is of class $C^{2}$ in this example and it shows that the sign of $\eta^{\prime \prime}(\alpha)$ may change. In virtue of the perturbation result of Section $3 \Gamma$ can be approximately reproduced by the classical boundary value problem (4).

\section{The linear equation}

Let $H_{0}^{1}=H_{0}^{1}(0,1)$ denote the usual space of real valued functions which have a generalised derivative in $L^{2}=L^{2}(0,1)$ and which vanish at $x=0$ and $x=1$. Then $H_{0}^{1}$ is a Hilbert space with respect to the scalar product $(u, v)=\int_{0}^{1} u^{\prime}(x) v^{\prime}(x) d x$

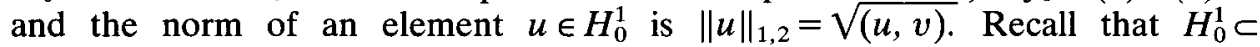
$C_{0}([0,1])$ is a continuous embedding and $\|u\|_{\infty}=\max _{x \in[0,1]}|u(x)| \leqq\|u\|_{1,2} \forall u \in H_{0}^{1}$. As 
usual the formula $\langle L u, v\rangle=(u, v)$ defines an isomorphism $L$ from $H_{0}^{1}$ into its dual $H^{-1}$ and $\|L\|=1$. Since there is an embedding $J: H_{0}^{1} \rightarrow H^{-1}$, defined by $\langle J u, v\rangle=\int_{0}^{1} u(x) v(x) d x \forall u, v \in H_{0}^{1}$, we can write an element $u$ of $H_{0}^{1}$ and the element $J u$ of $H^{-1}$ with the same symbol.

For $Q \in L^{2}$ define the generalised multiplication $M(Q): H_{0}^{1} \rightarrow H^{-1}$ by $\langle M(Q) u, v\rangle=-\int_{0}^{1}(u(x) v(x))^{\prime} Q(x) d x \forall u, v \in H_{0}^{1}$. Then $M(Q)$ is symmetric and bounded. In fact boundedness follows from

$$
|\langle M(Q) u, v\rangle|=2\left|\int_{0}^{1} u(x) u^{\prime}(x) Q(x) d x\right| \leqq 2\|u\|_{\infty}\|u\|_{1,2}\|Q\|_{2} \leqq 2\|u\|_{1,2}^{2}\|Q\|_{2} .
$$

For $Q \in L^{2}$ set $T(Q)=L+M(Q)$.

Lemma 2.1 (Gårding's inequality). For $Q \in L^{2}$ given, there exists $\lambda_{0} \in \mathbb{B}$ such that

$$
\langle T(Q) u, u\rangle-\lambda\langle u, u\rangle \geqq \frac{1}{2}\|u\|_{1,2}^{2} \forall \lambda \leqq \lambda_{0}, \forall u \in H_{0}^{1} .
$$

Proof. Let $Q \in L^{2}$ be given. For $u \in H_{0}^{1}$ recall that $\|u\|_{\infty}^{2} \leqq\|u\|_{2}\|u\|_{1,2}$ and then $|\langle M(Q) u, u\rangle| \leqq 2\|u\|_{2}^{\frac{1}{2}}\|u\|_{1,2}^{\frac{3}{2}}\|Q\|_{2}$. An application of Young's inequality implies that $\|u\|_{2}^{\frac{1}{2}}\|u\|_{1,2}^{\frac{1}{2}} \leqq \frac{1}{4} \alpha^{3}\|u\|_{2}^{2}+\frac{3}{4} \alpha^{-1}\|u\|_{1,2}^{2} \forall \alpha>0$ and consequently

$$
\begin{aligned}
\langle T(Q) u, u\rangle-\lambda\langle u, u\rangle & \geqq\langle L u, u\rangle-|\langle M(Q) u, u\rangle|-\lambda\langle u, u\rangle \\
& \geqq\left(1-\frac{3}{2} \alpha^{-1}\|Q\|_{2}\right)\|u\|_{1,2}^{2}-\left(\frac{1}{2} \alpha^{3}\|Q\|_{2}+\lambda\right)\langle u, u\rangle .
\end{aligned}
$$

Setting $\alpha=3\|Q\|_{2}, \lambda_{0}=-\frac{27}{2}\|Q\|_{2}^{4}$, the lemma is proved.

To state some properties of $T(Q)$ we prove the following.

Proposition 2.2. (a) The spectrum of $T(Q)$ consists of an increasing sequence of eigenvalues which tends to infinity.

(b) The eigenvalues of $T(Q)$ are simple.

Proof. (a) Let $\lambda_{0} \in \mathbb{R}$ be as in Lemma 2.1, fix $\lambda<\lambda_{0}$ and define the symmetric bilinear form $a: H_{0}^{1} \times H_{0}^{1} \rightarrow \mathbb{R}$ by $a(u, v)=\langle T(Q) u, v\rangle-\lambda\langle u, v\rangle$. Since $\frac{1}{2}\|u\|_{1,2}^{2} \leqq a(u, u) \leqq\|T(Q)-\lambda I\|\|u\|_{1,2}^{2}, a(.,$.$) is a scalar product on H_{0}^{1}$ and defines an equivalent norm of $\|\cdot\|_{1,2}$. In order to verify that $T(Q)-\lambda I: H_{0}^{1} \rightarrow H^{-1}$ is bijective let $\phi \in H^{-1}$ be given. By the Representation Theorem of RieszFréchet there exists a unique $u \in H_{0}^{1}$ such that $\langle\phi, v\rangle=a(u, v)=\langle T(Q) u, v\rangle-$ $\lambda\langle u, v\rangle$ for all $v \in H_{0}^{1}$. Hence the resolvent $(T(Q)-\lambda I)^{-1}: H^{-1} \rightarrow H^{-1}$ exists and is compact by the compact embedding $H_{0}^{1} \subset H^{-1}$. Thus the spectrum of $T(Q)$ consists of an increasing sequence of eigenvalues which tends to infinity.

(b) Let $\alpha$ be an eigenvalue of $T(Q)$ and let $u \in H_{0}^{1}$ be a corresponding eigenfunction. One has

$$
\int_{0}^{1} u^{\prime}(x) v^{\prime}(x) d x-\int_{0}^{1}(u(x) v(x))^{\prime} Q(x) d x=\alpha \int_{0}^{1} u(x) v(x) d x \forall v \in H_{0}^{1} .
$$

Integration by parts implies that

$$
\int_{0}^{1} v^{\prime}(x)\left[u^{\prime}(x)-u(x) Q(x)+\int_{0}^{x}\left(u^{\prime}(t) Q(t)+\alpha u(t)\right) d t\right] d x=0 \forall v \in H_{0}^{1} .
$$


Hence there exists a constant $c$ such that

$$
u^{\prime}(x)=u(x) Q(x)-\int_{0}^{x}\left(u^{\prime}(t) Q(t)+\alpha u(t)\right) d t+c \text { almost everywhere on }(0,1) .
$$

Let $u_{1}, u_{2}$ be two solutions of (7) and $c_{1}, c_{2}$ their respective constants. We set $w=c_{2} u_{1}-c_{1} u_{2}$ and show that $w \equiv 0$ on $[0,1]$. In fact $w$ satisfies

$$
w^{\prime}(x)=w(x) Q(x)-\int_{0}^{x}\left(w^{\prime}(t) Q(t)+\alpha w(t)\right) d t \text { almost everywhere on }(0,1) .
$$

Multiply this equation by $w^{\prime}(x)$ and integrate it over $(0, \varepsilon), 0<\varepsilon<1$, then

$$
\int_{0}^{\varepsilon} w^{\prime 2}(x) d x=\int_{0}^{\varepsilon} w(x) w^{\prime}(x) Q(x) d x-\int_{0}^{\varepsilon} w^{\prime}(x) \int_{0}^{x}\left(w^{\prime}(t) Q(t)+\alpha w(t)\right) d t d x .
$$

Note that $|w(x)| \leqq \int_{0}^{x}\left|w^{\prime}(t)\right| d t \leqq \sqrt{\varepsilon}\left\|w^{\prime}\right\|_{L^{2}(0, \varepsilon)}$ almost everywhere on $(0, \varepsilon)$ and estimate the terms on the right hand side:

$$
\begin{aligned}
&\left|\int_{0}^{\varepsilon} w(x) w^{\prime}(x) Q(x) d x\right| \leqq\|w\|_{L^{\infty}(0, \varepsilon)}\left\|w^{\prime}\right\|_{L^{2}(0, \varepsilon)}\|Q\|_{L^{2}(0, \varepsilon)} \\
& \leqq \sqrt{\varepsilon}\|Q\|_{L^{2}(0, \varepsilon)}\left\|w^{\prime}\right\|_{L^{2}(0, \varepsilon)}^{2} \\
&\left|\int_{0}^{\varepsilon} w^{\prime}(x) \int_{0}^{x} w^{\prime}(t) Q(t) d t d x\right| \leqq\left\|w^{\prime}\right\|_{L^{2}(0, \varepsilon)}\|Q\|_{L^{2}(0, \varepsilon)} \int_{0}^{\varepsilon}\left|w^{\prime}(x)\right| d x \\
& \leqq \sqrt{\varepsilon}\|Q\|_{L^{2}(0, \varepsilon)}\left\|w^{\prime}\right\|_{L^{2}(0, \varepsilon)}^{2} \\
&\left|\int_{0}^{\varepsilon} w^{\prime}(x) \int_{0}^{x} \alpha w(t) d t d x\right| \leqq \varepsilon|\alpha|\|w\|_{L^{\infty}(0, \varepsilon)} \int_{0}^{\varepsilon}\left|w^{\prime}(x)\right| d x \leqq \varepsilon^{2}|\alpha|\left\|w^{\prime}\right\|_{L^{2}(0, \varepsilon)}^{2} .
\end{aligned}
$$

Finally one obtains $\left\|w^{\prime}\right\|_{L^{2}(0, \varepsilon)}^{2} \leqq \sqrt{\varepsilon}\left(2\|Q\|_{2}+|\alpha|\right)\left\|w^{\prime}\right\|_{L^{2}(0, \varepsilon)}^{2}$ and for $\varepsilon_{0}=$ $\left(4\|Q\|_{2}+2|\alpha|\right)^{-2}$ we find that $w^{\prime}=0$ almost everywhere on $\left(0, \varepsilon_{0}\right)$. Hence $w \equiv 0$ on $\left(0, \varepsilon_{0}\right)$. Repeating the same argument for the intervals $\left(\varepsilon_{0}, 2 \varepsilon_{0}\right)$, $\left(2 \varepsilon_{0}, 3 \varepsilon_{0}\right), \ldots$ we deduce that $w \equiv 0$ on $[0,1]$. Thus $u_{1}$ and $u_{2}$ are linearly dependent and $\alpha$ is a simple eigenvalue.

The next lemma concerns the stability of the first eigenvalue of $T(Q)$, denoted by $\alpha(Q)$, under small perturbations of $Q \in L^{2}$.

Lemma 2.3. Given $P \in L^{2}$ and $\varepsilon>0$, there exists $\delta>0$ such that, if $Q \in L^{2}$ satisfies $\|P-Q\|_{2}<\delta$, then $|\alpha(P)-\alpha(Q)|<\varepsilon$.

Proof. By Lemma 2.1 there exists $\lambda \in \mathbb{R}$ such that $\|u\|_{1,2}^{2} \leqq 2\langle T(P) u, u\rangle-$ $2 \lambda\langle u, u\rangle$ for all $u \in H_{0}^{1}$, then by the minimal characterisation of the first eigenvalue

$$
\begin{aligned}
\alpha(Q) & =\min _{\|u\|_{2}=1}\langle T(Q) u, u\rangle=\min _{\|u\|_{2}=1}(\langle T(P) u, u\rangle+\langle M(Q-P) u, u\rangle) \\
& \leqq \min _{\|u\|_{2}=1}\left(\langle T(P) u, u\rangle+\|M(Q-P)\|\|u\|_{1,2}^{2}\right) \\
& \leqq \min _{\|u\|_{2}=1}\left(\langle T(P) u, u\rangle+4\|Q-P\|_{2}(\langle T(P) u, u\rangle-\lambda)\right) \\
& =\alpha(P)+4\|Q-P\|_{2}(\alpha(P)-\lambda) .
\end{aligned}
$$


Similarly $\alpha(Q) \geqq \alpha(P)-4\|Q-P\|_{2}(\alpha(P)-\lambda)$ and setting $\delta=\varepsilon / 4(\alpha(P)-\lambda)^{-1}$ the lemma is proved.

For $Q \in L^{2}$ and $l \in(0,1)$ consider the problem

$$
\int_{0}^{l} u^{\prime}(x) v^{\prime}(x) d x-\int_{0}^{l}(u(x) v(x))^{\prime} Q(x) d x=\alpha \int_{0}^{l} u(x) v(x) d x \forall v \in H_{0}^{1}(0, l) .
$$

Set $y=x / l, \hat{u}(y)=u(x), \hat{v}(y)=v(x)$ and define $Q_{l}(y)=Q(x)$, then (9) is equivalent to

$$
\int_{0}^{1} \hat{u}^{\prime}(y) \hat{v}^{\prime}(y) d y-\int_{0}^{1}(\hat{u}(y) \hat{v}(y))^{\prime} l Q_{l}(y) d y=l^{2} \alpha \int_{0}^{1} \hat{u}(y) \hat{v}(y) d y \forall \hat{v} \in H_{0}^{1} .
$$

Lemma 2.4. Let $P \in L^{2}, m \in(0,1)$ and $\varepsilon>0$ be given. There exist $\delta_{1}, \delta_{2}>0$ such that, if $Q \in L^{2}$ and $l \in(0,1)$ satisfy $\|P-Q\|_{2}<\delta_{1}$, respectively $|m-l|<\delta_{2}$, then $\left\|m P_{m}-l Q_{l}\right\|_{2}<\varepsilon$.

Proof. Let $P \in L^{2}, m \in(0,1)$ and $\varepsilon>0$ be given. Since $C([0,1])$ is densely embedded in $L^{2}$, for all $\varepsilon_{1}>0$ there exists $p \in C([0,1])$ such that $\|P-p\|_{2}<\varepsilon_{1}$. For $Q \in L^{2}$ and $l \in(0,1)$, by the triangle inequality,

$$
\begin{aligned}
\left\|m P_{m}-l Q_{l}\right\|_{2} \leqq m\left\|P_{m}-p_{m}\right\|_{2} & +m\left\|p_{m}-p_{l}\right\|_{2} \\
& +m\left\|p_{l}-P_{l}\right\|_{2}+m\left\|P_{l}-Q_{l}\right\|_{2}+\mid m-l\left\|Q_{l}\right\|_{2} .
\end{aligned}
$$

Note that $m\left\|P_{m}-p_{m}\right\|_{2} \leqq \sqrt{m}\|P-p\|_{2}<\varepsilon_{1}$ and $\left\|p_{m}-p_{l}\right\|_{2}<\varepsilon_{1}$ if $|m-l|$ is sufficiently small. Treating the other terms similarly, for $\varepsilon_{1}>0$ sufficiently small, there exist $\delta_{1}, \delta_{2}>0$ such that $\left\|m P_{m}-l Q_{l}\right\|_{2}<l \varepsilon$ whenever $\|P-Q\|_{2}<\delta_{1}$ and $|m-l|<\delta_{2}$.

THEOREM 2.5. Let $\alpha(l, Q)$ denote the first eigenvalue of $(9)$; then $A:(0,1) \times$ $L^{2} \rightarrow \mathbb{R}$ defined by $A(l, Q)=\alpha(l, Q)$ is continuous.

Theorem 2.5 is a direct consequence of formula (10), Lemma 2.3 and Lemma 2.4.

Lemma 2.6. Suppose that $c \in \mathbb{R}$ is given, then the first eigenfunction $u=$ $u(l, Q) \in H_{0}^{1}(0, l)$ of $(9)$ can be normalised such that

$u^{\prime}(x)=u(x) Q(x)-\int_{l}^{x}\left(u^{\prime}(t) Q(t)+\alpha(l, Q) u(t)\right) d t+c$ almost everywhere on $(0, l)$.

Furthermore $U:(0,1) \times L^{2} \rightarrow \mathbb{R}$ defined by $U(l, Q)=\|u(l, Q)\|_{2}$ is continuous.

Proof. Recall that a solution $u \in H_{0}^{1}(0, l)$ of (9) satisfies (11) for some $c \in \mathbb{R}$. If $u \neq 0$, then, proceeding as in the proof of Proposition 2.2(b), we conclude that $c \neq 0$. The linearity of the problem then implies that every constant $c \in \mathbb{R}$ can be achieved.

Setting $y=x / l, \hat{u}(y)=u(x)$ and $Q_{l}(y)=Q(x),(11)$ reduces to

$$
\begin{aligned}
\hat{u}^{\prime}(y)=\hat{u}(y) l Q_{l}(y) & -\int_{1}^{y}\left(\hat{u}^{\prime}(s) l Q_{l}(s)\right. \\
& \left.+l^{2} \alpha(l, Q) \hat{u}(s)\right) d s+l c \quad \text { almost everywhere on }(0,1) .
\end{aligned}
$$


Define the bounded linear functional $L_{l, Q}: H_{0}^{1} \rightarrow \mathbb{R}$ by

$$
L_{l, Q}(v)=\int_{0}^{1}\left[v(y) l Q_{l}(y)-\int_{1}^{y}\left(v^{\prime}(s) l Q_{l}(s)+l^{2} \alpha(l, Q) v(s)\right) d s\right] d y .
$$

Since the eigenspace of (9) is one-dimensional, the norm of the solution $\hat{u}(l, Q)$ is uniquely determined by $L_{l, Q}(\hat{u}(l, Q))+l c=0$. Fix $m \in(0,1)$ and $P \in L^{2}$, then by standard perturbation theory there exists $\hat{v}(l, Q) \in \operatorname{span}\{\hat{u}(l, Q)\}$ such that $\|\hat{v}(l, Q)-\hat{u}(m, P)\|_{1,2} \rightarrow 0$ as $|l-m| \rightarrow 0,\|Q-P\|_{2} \rightarrow 0$. One verifies that $\hat{u}(l, Q)=-c l \hat{v}(l, Q) / L_{l, Q}(\hat{v}(l, Q))$. By the continuity of $L_{l, Q}$ with respect to $l$ and $Q$ we have $\|\hat{u}(l, Q)\|_{1,2} \rightarrow\|\hat{u}(m, P)\|_{1,2}$ as $|l-m| \rightarrow 0,\|Q-P\|_{2} \rightarrow 0$. Passing to the original coordinates the lemma is proved.

\section{The nonlinear equation}

Let $\alpha(z, Q)$ and $\beta(z, Q)$ denote the first eigenvalues of (5) and (6) and let the corresponding eigenfunctions $u_{1} \in H_{0}^{1}(0, z)$ and $u_{2} \in H_{0}^{1}(z, 1)$ satisfy the normalisations

$$
\begin{aligned}
& \begin{aligned}
u_{1}^{\prime}(x)=u_{1}(x) Q(x)-\int_{z}^{x}\left(u_{1}^{\prime}(t) Q(t)\right. & \\
& \left.+\alpha(z, Q) u_{1}(t)\right) d t+c \text { almost everywhere on }(0, z)
\end{aligned} \\
& \begin{array}{r}
u_{2}^{\prime}(x)=u_{2}(x) Q(x)-\int_{z}^{x}\left(u_{2}^{\prime}(t) Q(t)\right. \\
\left.\quad+\beta(z, Q) u_{2}(t)\right) d t+c \text { almost everywhere on }(z, 1)
\end{array}
\end{aligned}
$$

where $c<0$ is a given number. Define

$$
u(x)=\left\{\begin{array}{lll}
u_{1}(x) & \text { for } & x \in[0, z] \\
u_{2}(x) & \text { for } & x \in(z, 1]
\end{array}\right.
$$

As in the proof of Proposition 2.2(b), there exists $K>0$ such that $\left\|u_{1}^{\prime}\right\|_{L^{2}(z-\varepsilon, z)}^{2} \leqq$ $\sqrt{\varepsilon} K\left\|u_{1}^{\prime}\right\|_{L^{2}(z-\varepsilon, z)}^{2}-c u_{1}(z-\varepsilon)$. Thus $u_{1}(z-\varepsilon)>0$ if $0<\varepsilon<K^{-2}$ and it follows that $u_{1}>0$ on $(0, z)$. In the same way one verifies that $u_{2}<0$ on $(z, 1)$. Since for all $v \in H_{0}^{1}$,

$$
\begin{gathered}
\int_{0}^{1} v^{\prime}(x)\left[u^{\prime}(x)-u(x) Q(x)+\int_{z}^{x}\left(u^{\prime}(t) Q(t)+\alpha(z, Q) u(t)^{+}-\beta(z, Q) u(t)^{-}\right) d t\right] d x \\
=\int_{0}^{1} v^{\prime}(x) c d x=0,
\end{gathered}
$$

then $u$ is a solution of (3) and therefore $\alpha(z, Q)$ and $\beta(z, Q)$ are parameters for (3) to have nontrivial solutions. By Theorem 2.5 the set $\Gamma(Q)$ defined by $\Gamma(Q)=\left\{(\alpha(z, Q), \beta(z, Q)) \in \mathbb{R}^{2}: z \in(0,1)\right\}$ is a continuous curve. Let $\lambda_{k}(Q)$, $k=1,2, \ldots$ denote the eigenvalues of $T(Q)$ and let $\psi_{k}(Q)$ be eigenfunctions of $T(Q)$ corresponding to $\lambda_{k}(Q)$ such that $\left\|\psi_{k}(Q)\right\|_{2}=1$ and $\psi_{2}(Q)(x)>0$ for $x$ near 0 . 
Proposition 3.1. If $(\alpha, \beta) \in \Gamma(Q)$, then (3) has an unique solution $u \in H_{0}^{1}$ such that

$$
\left\langle u, \psi_{2}(Q)\right\rangle=1 \text {. }
$$

Proof. Let $z_{0}$ be the unique zero of $\psi_{2}(Q), u \in H_{0}^{1}$ a solution of (3) with $u(x)>0$ for $x$ near 0 and let $z \in(0,1)$ denote the unique zero of $u$. Suppose first that $z_{0}<z<1$, then by the inclusions $H_{0}^{1}\left(0, z_{0}\right) \subset H_{0}^{1}(0, z), H_{0}^{1}(z, 1) \subset H_{0}^{1}\left(z_{0}, 1\right)$ and by the minimal characterisation of the first eigenvalue, $\alpha(z, Q)<\lambda_{2}(Q)<$ $\beta(z, Q)$. One has

$$
\begin{aligned}
\lambda_{2}(Q)\left\langle u, \psi_{2}(Q)\right\rangle & =\left\langle u, T(Q) \psi_{2}(Q)\right\rangle \\
& =\left\langle T(Q) u, \psi_{2}(Q)\right\rangle=\left\langle\alpha(z, Q) u^{+}-\beta(z, Q) u^{-}, \psi_{2}(Q)\right\rangle \\
& =\alpha(z, Q)\left\langle u, \psi_{2}(Q)\right\rangle+(\alpha(z, Q)-\beta(z, Q))\left\langle u^{-}, \psi_{2}(Q)\right\rangle .
\end{aligned}
$$

Hence

$$
\left\langle u, \psi_{2}(Q)\right\rangle=\frac{\alpha(z, Q)-\beta(z, Q)}{\lambda_{2}(Q)-\alpha(z, Q)}\left\langle u^{-}, \psi_{2}(Q)\right\rangle>0 .
$$

The case $0<z<z_{0}$ is handled in the same manner and the case $z=z_{0}$ is trivial. Thus $\left\langle u, \psi_{2}(Q)\right\rangle>0$ whenever $u(x)>0$ for $x$ near 0 and by the positive homogeneity of (3), (13) can be satisfied. Uniqueness follows from Proposition 2.2(b).

THeOrem 3.2. Let $P \in L^{2}, \varepsilon>0$ and $a, b \in(0,1), a<b$, be given. There exists $\delta>0$ such that, if $Q \in L^{2}$ satisfies $\|P-Q\|_{2}<\delta$, then for all $z \in[a, b]$, $\left((\alpha(z, P)-\alpha(z, Q))^{2}+(\beta(z, P)-\beta(z, Q))^{2}\right)^{\frac{1}{2}}<\varepsilon$.

Proof. Set

$$
\begin{aligned}
& \delta_{\alpha}(z)=\sup \left\{\gamma \in \mathbb{R}:|\alpha(z, P)-\alpha(z, Q)|<\frac{\sqrt{2} \varepsilon}{2} \forall Q \in L^{2} \text { such that }\|P-Q\|_{2}<\gamma\right\}, \\
& \delta_{\beta}(z)=\sup \left\{\gamma \in \mathbb{R}:|\beta(z, P)-\beta(z, Q)|<\frac{\sqrt{2} \varepsilon}{2} \forall Q \in L^{2} \text { such that }\|P-Q\|_{2}<\gamma\right\}
\end{aligned}
$$

and let us show that $\delta_{1}=\inf \left\{\delta_{\alpha}(z): z \in[a, b]\right\}>0$. Then likewise $\delta_{2}=$ $\inf \left\{\delta_{\beta}(z): z \in[a, b]\right\}>0$ and by setting $\delta=\min \left\{\delta_{1}, \delta_{2}\right\}$ the theorem is proved. By way of contradiction, suppose that $\delta_{\alpha}\left(z_{i}\right) \rightarrow 0$ for a sequence $\left(z_{i}\right) \subset[a, b]$. Then $z_{j} \rightarrow \hat{z} \in[a, b]$ for a subsequence. By Theorem 2.5 there exists $v>0$ such that $|\alpha(\hat{z}, P)-\alpha(z, Q)|<\sqrt{2} \varepsilon / 4$ for all $Q \in L^{2}$ such that $\|P-Q\|_{2}<v$ and for all $z \in(\hat{z}-v, \hat{z}+v)$. Consequently for those $z$ and $Q$

$$
|\alpha(z, Q)-\alpha(z, P)| \leqq|\alpha(z, Q)-\alpha(\hat{z}, P)|+|\alpha(\hat{z}, P)-\alpha(z, P)|<\frac{\sqrt{2} \varepsilon}{2} .
$$

Hence $\delta_{\alpha}(z) \geqq v>0$ for all $z \in(\hat{z}-v, \hat{z}+v)$. This is in contradiction to the assumption.

Remark 1. $\Gamma(Q)$ is unbounded on both sides. More precisely $\lim _{z \rightarrow 0^{+}} \alpha(z, Q)$ $=\infty$ and $\lim _{z \rightarrow 1^{-}} \beta(z, Q)=\infty$. In fact

$$
\lim _{z \rightarrow 0^{+}}\left\|z Q_{z}\right\|_{2}^{2}=\lim _{z \rightarrow 0^{+}} \int_{0}^{1} z^{2} Q(z x)^{2} d x=\lim _{z \rightarrow 0^{+}} z \int_{0}^{z} Q(y)^{2} d y=0,
$$


then by $(10)$ and Theorem $2.5 \lim _{z \rightarrow 0^{+}} z^{2} \alpha(z, Q)=\pi^{2}$. Thus $\lim _{z \rightarrow 0^{+}} \alpha(z, Q)=\infty$ and similarly $\lim _{z \rightarrow 1^{-}} \beta(z, Q)=\infty$.

Remark 2. Recall that $\alpha(., Q)$ is strictly decreasing, $\beta(., Q)$ is strictly increasing and $\lim _{z \rightarrow 1^{-}} \alpha(z, Q)=\lambda_{\mathrm{I}}(Q)$. Hence there exists a continuous and strictly decreasing function $\eta:\left(\lambda_{1}(Q), \infty\right) \rightarrow \mathbb{R}$ such that $\Gamma(Q)=\{(\alpha, \eta(\alpha)): \alpha \in$ $\left.\left(\lambda_{1}(Q), \infty\right)\right\}$.

THEOREM 3.3. There exist an open interval $J \subset\left(\lambda_{1}(Q), \lambda_{3}(Q)\right)$ and a $C^{1}$-function $\eta: J \rightarrow \mathbb{R}$ such that $\Gamma(Q) \cap\left(\lambda_{1}(Q), \lambda_{3}(Q)\right) \times\left(\lambda_{1}(Q), \lambda_{3}(Q)\right)=\{(\alpha, \eta(\alpha)): \alpha \in J\}$. If $u(\alpha, \beta) \in H_{0}^{1}$ denotes the solution of (3) and (13), then

$$
\eta^{\prime}(\alpha)=-\frac{\left\|u(\alpha, \eta(\alpha))^{+}\right\|_{2}^{2}}{\left\|u(\alpha, \eta(\alpha))^{-}\right\|_{2}^{2}} \text { for all } \alpha \in J .
$$

Proof. By the result of C. A. Stuart [4] the theorem is true for $Q \equiv 0$ and it generalises easily to $Q \in C^{1}([0,1])$. If $Q \in L^{2}$, then there exist $Q^{(n)} \in C^{1}([0,1])$, $n=1,2, \ldots$ such that $\left\|Q-Q^{(n)}\right\|_{2} \rightarrow 0$ as $n \rightarrow \infty$. By Remark 2 there exist open intervals $J \subset\left(\lambda_{1}(Q), \lambda_{3}(Q)\right), J_{n} \subset\left(\lambda_{1}\left(Q^{(n)}\right), \lambda_{3}\left(Q^{(n)}\right)\right)$ and continuous functions $\eta$ : $J \rightarrow \mathbb{R}, \quad \eta_{n}: J_{n} \rightarrow \mathbb{R}$ such that $\Gamma(Q) \cap\left(\lambda_{1}(Q), \lambda_{3}(Q)\right)^{2}=\{(\alpha, \eta(\alpha)): \alpha \in J\}$ and $\Gamma\left(Q^{(n)}\right) \cap\left(\lambda_{1}\left(Q^{(n)}\right), \lambda_{3}\left(Q^{(n)}\right)\right)^{2}=\left\{\left(\alpha, \eta_{n}(\alpha)\right): \alpha \in J_{n}\right\}, n=1,2, \ldots$ By [4] the $\eta_{n}$ are of class $C^{1}$. Let $u(\alpha, \eta(\alpha)), u_{n}\left(\alpha, \eta_{n}(\alpha)\right) \in H_{0}^{1}$ denote the solutions of $(3)$ and (13) for $Q$, respectively for $Q^{(n)}$; then

$$
\eta_{n}^{\prime}(\alpha)=-\frac{\left\|u_{n}\left(\alpha, \eta_{n}(\alpha)\right)^{+}\right\|_{2}^{2}}{\left\|u_{n}\left(\alpha, \eta_{n}(\alpha)\right)^{-}\right\|_{2}^{2}} \quad n=1,2, \ldots
$$

Note that $-\infty<\eta_{n}^{\prime}(\alpha)<0$ for all $\alpha \in J_{n}, n=1,2, \ldots$ Define the change of variables $\hat{\alpha}=1 / \sqrt{2}(\alpha-\beta), \hat{\beta}=1 / \sqrt{2}(\alpha+\beta)$; then for a $C^{1}$-function $\beta(\alpha)$, satisfying $-\infty<\beta^{\prime}(\alpha)<0$, the derivative transforms as follows:

$$
\hat{\beta}^{\prime}(\hat{\alpha})=\frac{1+\beta^{\prime}(\alpha)}{1-\beta^{\prime}(\alpha)} \text { and }\left|\hat{\beta}^{\prime}(\hat{\alpha})\right|<1 .
$$

Set $\hat{u}(\hat{\alpha})=u(\alpha, \eta(\alpha)), \hat{u}_{n}(\hat{\alpha})=u_{n}\left(\alpha, \eta_{n}(\alpha)\right), n=1,2, \ldots$, then $(14)$ is equivalent to

$$
\hat{\eta}_{n}^{\prime}(\hat{\alpha})=\frac{\left\|\hat{u}_{n}(\hat{\alpha})^{-}\right\|_{2}^{2}-\left\|\hat{u}_{n}(\hat{\alpha})^{+}\right\|_{2}^{2}}{\left\|\hat{u}_{n}(\hat{\alpha})\right\|_{2}^{2}} .
$$

Note that (14) does not depend upon the particular normalisation of $u_{n}$. Fix $c<0$ and normalise $u_{n}^{+}$by (11) for all $n$. Since $u_{n}^{-}$matches $u_{n}^{+}$, the $u_{n}^{-}$are normalised in the same way with the same constant $c$. By the Lebesque Dominated Convergence Theorem, Lemma 2.6 and Theorem 3.2

$$
\begin{aligned}
\hat{\eta}(\hat{\alpha})-\hat{\eta}\left(\hat{\alpha}_{0}\right) & =\lim _{n \rightarrow \infty}\left(\hat{\eta}_{n}(\hat{\alpha})-\hat{\eta}_{n}\left(\hat{\alpha}_{0}\right)\right)=\lim _{n \rightarrow \infty} \int_{\hat{\alpha}_{0}}^{\hat{\alpha}} \hat{\eta}_{n}^{\prime}(s) d s=\int_{\hat{\alpha}_{0}}^{\hat{\alpha}}\left(\lim _{n \rightarrow \infty} \hat{\eta}_{n}^{\prime}(s)\right) d s \\
& =\int_{\hat{\alpha}_{0}}^{\hat{\alpha}} \frac{\left\|\hat{u}(s)^{-}\right\|_{2}^{2}-\left\|\hat{u}(s)^{+}\right\|_{2}^{2}}{\|\hat{u}(s)\|_{2}^{2}} d s,
\end{aligned}
$$


for all $\hat{\alpha}, \hat{\alpha}_{0}$ such that $\alpha(\hat{\alpha}, \hat{\eta}(\hat{\alpha})), \alpha\left(\hat{\alpha}_{0}, \hat{\eta}\left(\hat{\alpha}_{0}\right)\right) \in J$. Again by Lemma 2.6 the integrand of the last formula is continuous and hence $\hat{\eta}$ is of class $C^{1}$. Differentiating $\hat{\eta}$ and passing to the original coordinates proves the theorem.

We summarise the results of this section in the following corollary using the notation of Theorem 3.3.

Corollary 3.4. Let $Q, Q^{(n)} \in L^{2}$ be such that $\left\|Q-Q^{(n)}\right\|_{2} \rightarrow 0$ as $n \rightarrow \infty$ and let $I \subset\left(\lambda_{1}(Q), \lambda_{3}(Q)\right)$ be a compact interval. There exists an integer $n_{0}$ such that $\left(\lambda_{1}\left(Q^{(n)}\right), \lambda_{3}\left(Q^{(n)}\right)\right) \supset I$ for all $n>n_{0}$ and $\lim _{n \rightarrow \infty}\left\|\eta-\eta_{n}\right\|_{C^{1}(I)}=0$.

\section{An example}

Given $a \in(0,1)$ and $p \in \mathbb{R}$, set

We have

$$
Q(x)=\left\{\begin{array}{lll}
0 & \text { for } & x \in[0, a] \\
p & \text { for } & x \in(a, 1] .
\end{array}\right.
$$

$$
\langle M(Q) u, v\rangle=-\int_{0}^{1}(u(x) v(x))^{\prime} Q(x) d x=p u(a) v(a) \text { for all } u, v \in H_{0}^{1},
$$

and we are concerned with the multiplication by the Dirac delta distribution up to the factor $p$. For $z \in[a, 1)$, (5) and (6) take the form

$$
\begin{gathered}
\int_{0}^{z} u^{\prime}(x) v^{\prime}(x) d x+p u(a) v(a)=\alpha \int_{0}^{z} u(x) v(x) d x \text { for all } v \in H_{0}^{1}(0, z) \\
\int_{z}^{1} u^{\prime}(x) v^{\prime}(x) d x=\beta \int_{z}^{1} u(x) v(x) d x \text { for all } v \in H_{0}^{1}(z, 1)
\end{gathered}
$$

and for $z \in(0, a]$ the situation is similar. We describe the set $\Gamma(Q)=$ $\left\{(\alpha(z, Q), \beta(z, Q)) \in \mathbb{R}^{2}: z \in(0,1)\right\}$ near the value $z=a$. We fix $p$ for the time being, omit the variable $Q$ and set $\alpha(z)=\alpha(z, Q)$ and $\beta(z)=\beta(z, Q)$. It is easily seen that $\alpha(a)=\pi^{2} / a^{2}$, thus by the continuity of $\alpha(\cdot), \alpha(z)>0$ for $z$ near $a$. Hence the first eigenfunction of (15) must take the form

$$
f(x)=\left\{\begin{array}{lll}
\sin \sqrt{\alpha} x & \text { for } & x \in[0, a] \\
\frac{\sin \sqrt{\alpha} a}{\sin \sqrt{\alpha}(z-a)} \sin \sqrt{\alpha}(z-x) & \text { for } & x \in(a, z] .
\end{array}\right.
$$

To determine the dependence of $\alpha$ on $z$ and $p$, note that by (8)

$$
f^{\prime}\left(a^{+}\right)-f^{\prime}\left(a^{-}\right)-p f(a)=0,
$$

or

$$
-\sqrt{\alpha} \sin \sqrt{\alpha} a \operatorname{ctg} \sqrt{\alpha}(z-a)-\sqrt{\alpha} \cos \sqrt{\alpha} a-p \sin \sqrt{\alpha} a=0 .
$$

For $(\alpha, z)$ near $\left(\pi^{2} / a^{2}, a\right)$ this relation is equivalent to finding zeros of

$$
F(\alpha, z)=(p \operatorname{tg} \sqrt{\alpha} a+\sqrt{\alpha}) \operatorname{tg} \sqrt{\alpha}(z-a)+\sqrt{\alpha} \operatorname{tg} \sqrt{\alpha} a .
$$


Note that $F\left(\pi^{2} / a^{2}, a\right)=0$. Let $F_{\alpha}, F_{z}, F_{\alpha z}, \ldots$ denote the partial derivatives of $F$. One verifies that

$$
F_{\alpha}\left(\frac{\pi^{2}}{a^{2}}, a\right)=\frac{a}{2} \text { and } F_{z}\left(\frac{\pi^{2}}{a^{2}}, a\right)=\frac{\pi^{2}}{a^{2}} .
$$

By the Implicit Function Theorem there exists a $C^{1}$-function $\phi(z)$ such that $\phi(a)=\pi^{2} / a^{2}$ and $F(\phi(z), z)=0$ for $z$ near $a$. In particular $\alpha(z)=\phi(z)$ for $z \geqq a$ and $\alpha^{\prime}\left(a^{+}\right)=\phi^{\prime}(a)=-2 \pi^{2} / a^{3}$. Concerning (16), one has $\beta(z)=\pi^{2} /(1-z)^{2}$ for $z \in[a, 1)$. By Theorem 3.3 there exists an open interval $J \subset \mathbb{P}$ and a $C^{1}$-function $\eta: J \rightarrow \mathbb{R}$ such that $\Gamma(Q) \cap\left(\lambda_{1}(Q), \lambda_{3}(Q)\right)^{2}=\{(\alpha, \eta(\alpha)): \alpha \in J\}$; that is to say $\eta(\alpha(z))=\beta(z)$ for $z \in[a, 1)$. In particular

$$
\eta\left(\frac{\pi^{2}}{a^{2}}\right)=\beta(a)=\frac{\pi^{2}}{(1-a)^{2}} \quad \text { and } \quad \eta^{\prime}\left(\frac{\pi^{2}}{a^{2}}\right)=\beta^{\prime}\left(a^{+}\right)\left(\alpha^{\prime}\left(a^{+}\right)\right)^{-1}=-\left(\frac{a}{1-a}\right)^{3} .
$$

Let us turn to the second derivatives. From $F_{\alpha} \phi^{\prime}+F_{z}=0$ we deduce that

or

$$
F_{\alpha \alpha} \phi^{\prime 2}+2 F_{z \alpha} \phi^{\prime}+F_{z z}+F_{\alpha} \phi^{\prime \prime}=0,
$$

$$
\frac{\phi^{\prime \prime}}{\phi^{\prime 3}}=F_{z}^{-1}\left[F_{\alpha \alpha}+2 F_{z \alpha}\left(\phi^{\prime}\right)^{-1}+F_{z z}\left(\phi^{\prime}\right)^{-2}\right]
$$

By straightforward calculation

$$
F_{\alpha \alpha}\left(\frac{\pi^{2}}{a^{2}}, \alpha\right)=\frac{a^{3}}{4 \pi^{2}}, \quad F_{z \alpha}\left(\frac{\pi^{2}}{a^{2}}, a\right)=\frac{a p}{2}+1, \quad F_{z z}\left(\frac{\pi^{2}}{a^{2}}, a\right)=0 .
$$

Consequently

$$
\begin{aligned}
\eta^{\prime \prime}\left(\left(\frac{\pi^{2}}{a^{2}}\right)^{-}\right) & =\beta^{\prime \prime}\left(a^{+}\right)\left(\phi^{\prime}(a)\right)^{-2}-\beta^{\prime}\left(a^{+}\right) \phi^{\prime \prime}(a)\left(\phi^{\prime}(a)\right)^{-3} \\
& =\frac{6 \pi^{2}}{(1-a)^{4}} \frac{a^{6}}{4 \pi^{4}}+\frac{2 \pi^{2}}{(1-a)^{3}} \frac{a^{5}}{\pi^{4}}\left(\frac{3}{4}+\frac{a p}{2}\right) \\
& =\frac{3 a^{5}}{2 \pi^{2}(1-a)^{4}}+\frac{a^{6} p}{\pi^{2}(1-a)^{3}} .
\end{aligned}
$$

To calculate the limit at the right, recall the identity $\eta^{\prime \prime}(\alpha)=-\left(\eta^{\prime}(\alpha)\right)^{3} \times$ $\left(\eta^{-1}\right)^{\prime \prime}(\eta(\alpha))$ where $\eta^{-1}$ denotes the inverse of $\eta$. By a symmetry argument it is easily seen that $\left(\eta^{-1}\right)^{\prime \prime}\left(\left(\pi^{2} /(1-a)^{2}\right)^{-}\right)$is obtained from $\eta^{\prime \prime}\left(\left(\pi^{2} / a^{2}\right)^{-}\right)$replacing $a$ by $1-a$. Hence

$$
\begin{aligned}
\eta^{\prime \prime}\left(\left(\frac{\pi^{2}}{a^{2}}\right)^{+}\right) & =-\eta^{\prime}\left(\frac{\pi^{2}}{a^{2}}\right)^{3}\left(\eta^{-1}\right)^{\prime \prime}\left(\left(\frac{\pi^{2}}{(1-a)^{2}}\right)^{-}\right) \\
& =\left(\frac{a}{1-a}\right)^{9}\left[\frac{3(1-a)^{5}}{2 \pi^{2} a^{4}}+\frac{(1-a)^{6} p}{\pi^{2} a^{3}}\right]=\eta^{\prime \prime}\left(\left(\frac{\pi^{2}}{a^{2}}\right)^{-}\right) .
\end{aligned}
$$

This implies that $\eta$ is of class $C^{2}$ near $\alpha=\pi^{2} / a^{2}$ and

$$
\eta^{\prime \prime}\left(\frac{\pi^{2}}{a^{2}}\right)=\frac{3 a^{5}}{2 \pi^{2}(1-a)^{4}}+\frac{a^{6} p}{\pi^{2}(1-a)^{3}} .
$$




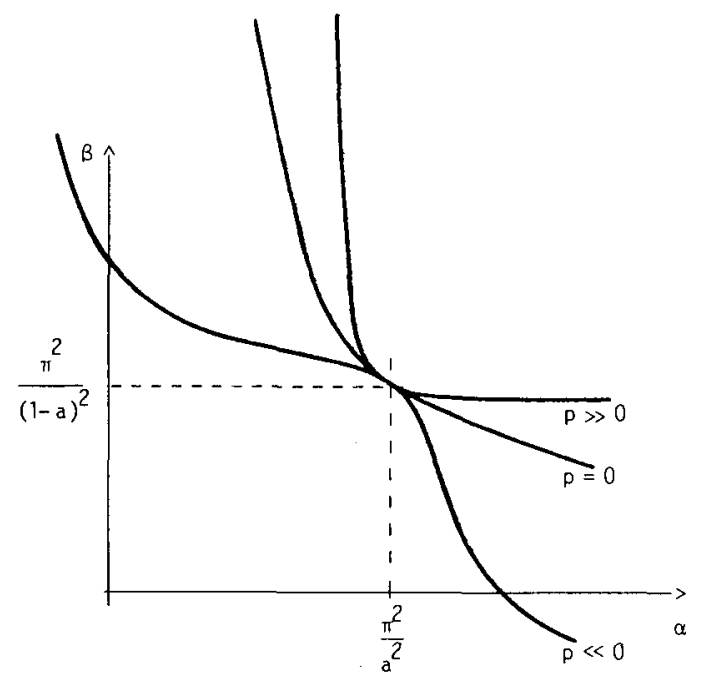

Figure 1

The second term of this formula is the contribution of the Dirac distribution. Note that $\eta^{\prime \prime}\left(\pi^{2} / a^{2}\right)>0$ if and only if $p>-\frac{3}{2 a(1-a)}$, which for $\Gamma(Q)$ yields the
diagram in Figure 1 .

Finally we investigate $\Gamma(Q)$ under the limits $p \rightarrow \pm \infty$. Fix $z_{0} \in(a, 1)$ and define $\alpha_{0}(p)=\alpha\left(z_{0}, Q\right), \beta_{0}(p)=\beta\left(z_{0}, Q\right)$. Note that $\beta_{0}$ is constant, $\alpha_{0}$ is continuous and strictly increasing. It is easy to see that $\alpha_{0}(p)<0$ for sufficiently negative $p$. In this case the solution of (15) takes the form

$$
f(x)= \begin{cases}\operatorname{sh} \sqrt{-\alpha} x & \text { for } \quad x \in[0, a] \\ \frac{\operatorname{sh} \sqrt{-\alpha} a}{\operatorname{sh} \sqrt{-\alpha}\left(z_{0}-a\right)} \operatorname{sh} \sqrt{-\alpha}\left(z_{0}-x\right) & \text { for } x \in\left(a, z_{0}\right] .\end{cases}
$$

By (17),

$$
-\sqrt{-\alpha} \operatorname{sh} \sqrt{-\alpha} a \text { th } \sqrt{-\alpha}\left(z_{0}-a\right)-\sqrt{-\alpha} \operatorname{ch} \sqrt{-\alpha} a-p \operatorname{sh} \sqrt{-\alpha} a=0
$$

and it follows that $\lim _{p \rightarrow-\infty} \alpha_{0}(p)=-\infty$. On the other hand $\alpha_{0}(p)>0$ for large $p$, then again by (17)

$$
-\sqrt{\alpha} \operatorname{ctg} \sqrt{\alpha}\left(z_{0}-a\right)-\sqrt{\alpha} \operatorname{ctg} \sqrt{\alpha} a-p=0 .
$$

Thus by the monotony of $\alpha_{0}, \lim _{p \rightarrow \infty} \alpha_{0}(p)=\min \left\{\frac{\pi^{2}}{\left(z_{0}-a\right)^{2}}, \frac{\pi^{2}}{a^{2}}\right\}$.

\section{Acknowledgment}

The author thanks Professor C. A. Stuart for his advice and encouragement during this work. 


\section{References}

1 T. Gallouët and $\mathrm{O}$. Kavian. Résultats d'existence et de non-existence de solutions pour certains problèmes demi-linéaires à l'infini. C.R. Acad. Sci. Paris 291 (1980), 193-196.

2 T. Gallouët and $O$. Kavian. Résultats d'existence et de non-existence pour certains problèmes demi-linéaires à l'infini. Ann. Fac. Sci. Toulouse Math. 3 (1981), 201-246.

3 B. Ruf. On nonlinear elliptic problems with jumping nonlinearities. Ann. Mat. Pura Appl. (4) 128 (1981), 133-151.

4 C. A. Stuart. Elliptic boundary-value problems with jumping nonlinearities. Rapport interne, Département de Mathématiques, Ecole Polytechnique Fédérale de Lausanne, CH-1015 Lausanne, Switzerland.

(Issued 21 December 1987) 